\title{
A GENERAL THEORY OF NETS ON A SURFACE*
}

\author{
BY \\ VERNON G. GROVE \\ INTRODUCTION
}

In this paper we extend a method used by E. P. Lane in the study of conjugate nets $\dagger$ to the study of a non-conjugate, non-asymptotic net on a surface. We first refer the sustaining surface to the asymptotic net, and then refer it to an arbitrary net. We thus find that all of the projective properties of the net are expressible in terms of the quantities determining the character of the surface and two other functions. These two functions may be chosen arbitrarily, and hence they determine the most general net on the surface. It may be seen, from the form of the formulas involved, which properties of the net are really properties of the net, which are properties of the one-parameter families of curves forming the net, and which are properties entirely of the surface.

\section{A SURFACE REFERRED TO ITS ASYMPTOTIC NET}

Let

$$
y^{(k)}=y^{(k)}(u, v) \quad(k=1,2,3,4)
$$

be the parametric equations of a surface $S_{y}$. If $S_{y}$ is not a developable surface, and if the curves $u=$ const., $v=$ const. are the asymptotic curves, the four functions $y^{(k)}$ are solutions of two partial differential equations of the form

$$
\begin{aligned}
& y_{u u}+2 a y_{u}+2 b y_{v}+c y=0, \\
& y_{v v}+2 a^{\prime} y_{u}+2 b^{\prime} y_{v}+c^{\prime} y=0 .
\end{aligned}
$$

These two equations can be reduced to the form $\ddagger$

$$
\begin{aligned}
& y_{u u}+2 b y_{v}+f y=0, \\
& y_{v v}+2 a^{\prime} y_{u}+g y=0 .
\end{aligned}
$$

* Presented to the Society, April 10, 1925; received by the editors in November, 1926.

† E. P. Lane, A general theory of conjugate nets, these Transactions, vol. 23 (1922), pp. 283-297. Cited as Lane, Nets.

$\ddagger$ E. J. Wilczynski, Projective differential geometry of curved surfaces, 1st memoir, these Transactions, vol. 8 (1907), p. 233. 
The coefficients of system (2) satisfy the following integrability conditions:

$$
\begin{aligned}
& a_{u u}^{\prime}+g_{u}+2 a_{v}^{\prime} b+4 a^{\prime} b_{v}=0, \\
& b_{v v}+f_{v}+2 a^{\prime} b_{u}+4 a_{u}^{\prime} b=0, \\
& g_{u u}+4 b_{v} g+2 b g_{v}=f_{v v}+4 a_{u}^{\prime} f+2 a^{\prime} f_{u} .
\end{aligned}
$$

The form (2) is not unique, but is preserved under all transformations of the form

$$
\alpha=U(u), \beta=V(v), \bar{y}=C\left(U^{\prime} V^{\prime}\right)^{1 / 2} y .
$$

Any net on the surface may be defined by a differential equation of the form

$$
(\theta d u-d v)(\omega d v-d u)=0, \quad 1-\theta \omega \neq 0 .
$$

If $1-\theta \omega$ were zero, the differential equation would represent only a oneparameter family of curves, and not a net. If $1+\theta \omega=0$, the net is a conjugate net. If $\theta=\omega=0$ the net is the asymptotic net. We shall suppose, therefore, that

$$
\theta \omega\left(1-\theta^{2} \omega^{2}\right) \neq 0 .
$$

2. THE SURFACE REFERRED to ANY NON-CONJUGATE NET

Let us make the net (3) parametric by the transformation

$$
\alpha=\phi(u, v), \quad \beta=\psi(u, v),
$$

for which

$$
\phi_{u}=-\theta \phi_{v}, \quad \psi_{v}=-\omega \psi_{u} .
$$

If we make this transformation we obtain the system of differential equations of the form

$$
\begin{aligned}
& y_{\alpha \alpha}=\bar{a} y_{\alpha \beta}+\bar{b} y_{\alpha}+\bar{c} y_{\beta}+\bar{d} y, \\
& y_{\beta \beta}=\bar{a} y_{\alpha \beta}+\bar{b}^{\prime} y_{\alpha}+\bar{c}^{\prime} y_{\beta}+\bar{d}^{\prime} y,
\end{aligned}
$$

wherein

$$
\begin{aligned}
\bar{a} & =\frac{2 \omega}{1+\theta \omega} \frac{\psi_{u}}{\phi_{v}}, \quad \bar{a}^{\prime}=\frac{2 \theta}{1+\theta \omega} \frac{\phi_{v}}{\psi_{u}} \\
\bar{b} & =-\frac{\phi_{v v}}{\phi_{v}^{2}}+\frac{1}{\phi_{v}\left(1-\theta^{2} \omega^{2}\right)}\left[2 b \omega^{2}+2 a^{\prime} \theta+\left(\theta \theta_{v}-\theta_{u}\right) \omega^{2}\right], \\
\bar{c}^{\prime} & =-\frac{\psi_{u u}}{\psi_{u}^{2}}+\frac{1}{\psi_{u}\left(1-\theta^{2} \omega^{2}\right)}\left[2 a^{\prime} \theta^{2}+2 b \omega+\left(\omega \omega_{u}-\omega_{v}\right) \theta^{2}\right],
\end{aligned}
$$




$$
\begin{aligned}
& \bar{c}=-\frac{\psi_{u}}{\phi_{v}^{2}\left(1-\theta^{2} \omega^{2}\right)}\left(2 b \omega^{3}+2 a^{\prime}+\omega \omega_{u}-\omega_{v}\right), \\
& \bar{b}^{\prime}=-\frac{\phi_{v}}{\psi_{u}^{2}\left(1-\theta^{2} \omega^{2}\right)}\left(2 a^{\prime} \theta^{3}+2 b+\theta \theta_{v}-\theta_{u}\right), \\
& \bar{d}=\frac{g-f \omega^{2}}{\psi_{u}^{2}\left(\theta^{2} \omega^{2}-1\right)}, \quad \bar{d}^{\prime}=\frac{f-g \theta^{2}}{\psi_{u}^{2}\left(\theta^{2} \omega^{2}-1\right)} .
\end{aligned}
$$

System (6) defines the same surface as system (2), but the parametric net for (6) is the arbitrary non-conjugate net (3).

The following differentiation formulas will be found useful:

$$
y_{\alpha}=\frac{\omega y_{u}+y_{v}}{(1-\theta \omega) \phi_{v}}, \quad y_{\beta}=\frac{y_{u}+\theta y_{v}}{(1-\theta \omega) \psi_{u}} .
$$

For convenience of reference we will give certain invariants and covariants of system (6). They are

$$
\begin{gathered}
\mathfrak{B}=\bar{a}^{\prime} \bar{\gamma}-\frac{1}{2}\left(f_{\beta}-2 \bar{c}^{\prime}\right), \quad \mathfrak{S}=\bar{a} \bar{\beta}^{\prime}-\frac{1}{2}\left(f_{\alpha}-2 \bar{b}\right)^{*}, \\
\rho=y_{\alpha}-\bar{\gamma} y, \quad \sigma=y_{\beta}-\bar{\beta}^{\prime} y \dagger,
\end{gathered}
$$

wherein

$$
\begin{gathered}
\bar{\gamma}=-\frac{\bar{c}}{\bar{a}}, \quad \bar{\beta}^{\prime}=-\frac{\bar{b}^{\prime}}{\bar{a}^{\prime}}, \\
f_{\alpha}=\bar{b}+\frac{1}{1-\bar{a} \bar{a}^{\prime}}\left[\bar{a}_{\beta}+\bar{b}+\bar{a}^{\prime} \bar{c}+\bar{a}\left(\bar{a}_{\alpha}^{\prime}+\bar{a} \bar{b}^{\prime}+\bar{c}^{\prime}\right)\right] \\
f_{\beta}=\bar{c}^{\prime}+\frac{1}{1-\bar{a} \bar{a}^{\prime}}\left[\bar{a}_{\alpha}^{\prime}+\bar{c}^{\prime}+\bar{a} \bar{b}^{\prime}+\bar{a}^{\prime}\left(\bar{a}_{\beta}+\bar{a}^{\prime} \bar{c}+\bar{b}\right)\right] .
\end{gathered}
$$

The axis of the point $y$ with respect to the net (3) joins $y$ to the point $\zeta$ defined by

$$
\zeta=y_{\alpha \beta}-\bar{\beta}^{\prime} y_{\alpha}-\bar{\gamma} y_{\beta} .
$$

Substituting the values of the coefficients (7) into (9) and (10) we obtain these expressions in terms of $\theta, \omega$, and the coefficients and variables of system (2). They are

* V. G. Grove, $A$ theory of a general net on a surface, these Transactions, vol. 28 (1926), p. 496. Hereafter referred to as Grove, Nets.

† G. M. Green, Nets of space curves, these Transactions, vol. 21 (1920), p. 219. Hereafter referred to as Green, Nets.

¥ Green, Nets, p. 224. 


$$
\begin{aligned}
\mathfrak{B}= & \frac{1}{\omega(1-\theta \omega)^{2}(1+\theta \omega) \psi_{u}}\left[2\left(a^{\prime} \theta+b \omega^{2}\right)\left(1-\theta^{2} \omega^{2}\right)-\omega(2+\theta \omega)(\theta \omega)_{u}\right. \\
\mathfrak{S}^{\prime}= & \frac{1}{\theta(1-\theta \omega)^{2}(1+\theta \omega) \phi_{v}}\left[2\left(b \omega+a^{\prime} \theta^{2}\right)\left(1-\theta^{2} \omega^{2}\right)-\theta(2+\theta \omega)(\theta \omega)_{v}\right. \\
\rho= & \frac{1}{(1-\theta \omega) \phi_{v}}\left[\omega y_{u}+y_{v}-\frac{1}{2 \omega}\left(2 b \omega^{3}+2 a^{\prime}+\omega \omega_{u}-\omega_{v}\right) y\right], \\
\sigma & =\frac{1}{(1-\theta \omega) \psi_{u}}\left[y_{u}+\theta y_{v}-\frac{1}{2 \theta}\left(2 a^{\prime} \theta^{3}+2 b+\theta \theta_{v}-\theta_{u}\right) y\right], \\
\zeta & =\frac{1+\theta \omega}{2 \theta \omega(1-\theta \omega)^{3} \phi_{v} \psi_{u}}\left\{2 \theta \omega(1-\theta \omega) y_{u v}-\left[2 a^{\prime} \theta\left(1-\theta^{2} \omega^{2}\right)+2 b \omega^{2}(1-\theta \omega)\right.\right. \\
& \left.-\omega(\theta \omega)_{u}-(\theta \omega)_{v}+\omega \theta_{v}(1-\theta \omega)\right] y_{u}-\left[2 b \omega\left(1-\theta^{2} \omega^{2}\right)+2 a^{\prime} \theta^{2}(1-\theta \omega)\right. \\
& \left.\left.-\theta(\theta \omega)_{v}-(\theta \omega)_{u}+\theta \omega_{u}(1-\theta \omega)\right] y_{v}+() y\right\},
\end{aligned}
$$

the coefficient of $y$ being immaterial for our purposes.

\section{THE $R$-RECIPROCAL CONGRUENCES}

Two congruences of importance in the theory of nets of curves are those congruences we have called the $R$-reciprocal congruences. ${ }^{*}$ The lines of these congruences are reciprocal polars with respect to both of the quadrics osculating the parametric ruled surfaces of tangents at the surface point $y$. The lines of these congruences are also in relation $R$. The lines $l$ and $l^{\prime}$ of the $R$-reciprocal congruences join the points

$$
r=y_{\alpha}-\frac{1}{2 \bar{a}^{\prime}}\left(f_{\beta}-2 \bar{c}^{\prime}\right) y, \quad s=y_{\beta}-\frac{1}{2 \bar{a}}\left(f_{\alpha}-2 \bar{b}\right) y,
$$

and

$$
y, z=y_{\alpha \beta}-\frac{1}{2 \bar{a}}\left(f_{\alpha}-2 \bar{b}\right) y_{\alpha}-\frac{1}{2 \bar{a}^{\prime}}\left(f_{\beta}-2 \bar{c}^{\prime}\right) y_{\beta} .
$$

The line $l$ intersects the asymptotic tangents in the two points defined by

$$
\begin{aligned}
r_{1}=y_{u}-\frac{1}{2 \theta \omega(1-\theta \omega)}\left[2 a^{\prime} \theta^{2}(\theta \omega-1)+\theta \omega(\theta \omega)_{u}\right. & +2 \theta(\theta \omega)_{v} \\
& \left.-\theta \omega_{u}(\theta \omega-1)\right] y, \\
s_{1}=y_{v}-\frac{1}{2 \theta \omega(1-\theta \omega)}\left[2 b \omega^{2}(\theta \omega-1)+\theta \omega(\theta \omega)_{v}\right. & +2 \omega(\theta \omega)_{u} \\
& \left.-\omega \theta_{v}(\theta \omega-1)\right] y .
\end{aligned}
$$

* Grove, Nets, p. 497. 
The expression for $\mathbf{z}$ in terms of the coefficients and variables of system (2) is

$$
\begin{aligned}
z=\frac{4 \theta \omega}{(1-\theta \omega) \phi_{v} \psi_{u}}\left\{y_{u v}\right. & -\left[\frac { 1 } { 2 \theta \omega ( 1 - \theta \omega ) } \left(2 b \omega^{2}(\theta \omega-1)+\theta \omega(\theta \omega)_{v}\right.\right. \\
+ & \left.\left.2 \omega(\theta \omega)_{u}-\omega \theta_{v}(\theta \omega-1)\right)+\frac{(\theta \omega)_{v}}{1-\theta^{2} \omega^{2}}\right] y_{u} \\
& -\left[\frac { 1 } { 2 \theta \omega ( 1 - \theta \omega ) } \left(2 a^{\prime} \theta^{2}(\theta \omega-1)+\theta \omega(\theta \omega)_{u}\right.\right. \\
+ & \left.\left.\left.2 \theta(\theta \omega)_{v}-\theta \omega_{u}(\theta \omega-1)\right)+\frac{(\theta \omega)_{u}}{1-\theta^{2} \omega^{2}}\right] y_{v}+() y\right\},
\end{aligned}
$$

the coefficient of $y$ being immaterial for our purposes.

Now the two lines joining the points

$$
r=y_{u}-\lambda y, \quad s=y_{v}-\mu y,
$$

and the point $y$ to

$$
z=y_{u v}-m y_{u}-n y_{v}
$$

are Green reciprocal lines* if, and only if, $m=\mu$ and $n=\lambda$. We note from (14) and (15) that the $R$-reciprocal congruences are also Green reciprocal congruences if, and only if, $\theta \omega=$ const.

From the first two of equations (7) we find

$$
\bar{a} \bar{a}^{\prime}=\frac{4 \theta \omega}{(1+\theta \omega)^{2}} .
$$

Hence if $\theta \omega$ is constant then $\bar{a} \bar{a}^{\prime}$ is constant, and conversely. If $\bar{a} \bar{a}^{\prime}=$ const. the tangents to the curves of the net form with the asymptotic tangents a constant cross ratio. $\dagger$ We may state our results in the following way: The R-reciprocal congruences are Green reciprocal congruences if and only if the tangents to the curves of the net form with the asymptotic tangents a constant cross ratio.

Let us now find the condition that the $R$-reciprocal congruences coincide with a given pair of Green reciprocal congruences. We must have $\theta \omega=$ const. Let the given lines join the points

* G. M. Green, Memoir on the general theory of surfaces and rectilinear congruences, these Transactions, vol. 20 (1919), p. 86. Cited as Green, Surfaces.

$\dagger$ Grove, Nets, p. 500. This result was also derived by E. P. Lane in his paper Bundles and pencils of nets on a surface, these Transactions, vol. 28 (1926), p. 165. 


$$
r=y_{u}-\lambda y, \quad s=y_{v}-\mu y,
$$

and $y$ to the point

$$
z=y_{u v}-\mu y_{u}-\lambda y_{v} .
$$

From (14) and (15) we have

$$
\theta \omega_{u}-2 a^{\prime} \theta^{2}=2 \theta \omega \lambda, \omega \theta_{v}-2 b \omega^{2}=2 \theta \omega \mu .
$$

Putting $\theta \omega=k$, where $k$ is a constant, these may be written

$$
\frac{\theta_{u}}{\theta}=-\frac{2 a^{\prime} \theta^{2}}{k}-2 \lambda, \quad \frac{\theta_{v}}{\theta}=\frac{2 b k}{\theta^{2}}+2 \mu .
$$

A necessary and sufficient condition that (16) have a solution is that the equation

$$
\frac{\partial}{\partial u}\left(\frac{b k}{\theta^{2}}+\mu\right)=\frac{\partial}{\partial v}\left(-\frac{a^{\prime} \theta^{2}}{k}-\lambda\right)
$$

be satisfied by such a solution. This condition of integrability may be written

$$
\left(a_{v}^{\prime}+4 a^{\prime} \mu\right) \theta^{4}+k\left(\mu_{u}+\lambda_{v}+8 a^{\prime} b\right) \theta^{2}+k^{2}\left(b_{u}+4 b \lambda\right)=0 .
$$

Two cases are possible:

1. This equation may be an identity in $\theta$, in which case the coefficients of the quadratic in $\theta^{2}$ are all zero.

2. The biquadratic may be solved for $\theta$.

In Case 1, we will assume that the surface is not ruled. Then

$$
\lambda=-\frac{b_{u}}{4 b}, \quad \mu=-\frac{a_{v}^{\prime}}{4 a^{\prime}}, \quad 8 a^{\prime} b+\mu_{u}+\lambda_{v}=0 .
$$

Hence the assigned pair of Green reciprocal congruences is not arbitrary; the pair must be the canonical congruences as defined by Green.* The third equation of (18) shows that the surface itself is restricted. This equation may be written

$$
\frac{\partial^{2}}{\partial u \partial v} \log \left(a^{\prime} b\right)=32 a^{\prime} b .
$$

If we put $a^{\prime} b=e^{\phi}$, equation (19) may be written

$$
\frac{\partial^{2} \phi}{\partial u \partial v}=32 e^{\phi} \text {. }
$$

\footnotetext{
* Green, Surfaces, p. 114
} 
The solution of (20) determines $\phi$ and hence the class of surfaces for which (19) holds. Since the solution of the completely integrable system (16) involves one arbitrary constant, we may state that for each surface of the type defined by equation (19) the canonical congruences of the first and second kind will serve as the $R$-reciprocal congruences for a two-pararieter family of nets on that surface.

In Case 2, the surface may be arbitrary, the given pair of congruences being restricted. If we solve the biquadratic for $\theta$ and substitute the values of $\theta$ in equations (16), there result two differential equations of the second order which $\lambda$ and $\mu$ must satisfy. These equations are of the form

$$
\begin{aligned}
& \lambda_{u v}+\mu_{u u}=G\left(\lambda, \mu, \lambda_{u}, \mu_{u}, \lambda_{v}, a^{\prime}, b\right), \\
& \lambda_{v v}+\mu_{u v}=G^{\prime}\left(\lambda, \mu, \lambda_{v}, \mu_{v}, \mu_{u}, a^{\prime}, b\right),
\end{aligned}
$$

where $G$ and $G^{\prime}$ are explicit functions of the indicated arguments. No restrictions are required for a solution of (21). Hence in Case 2, there exists for any surface $S_{y}$ whatever, a class of Green reciprocal congruences defined by (21), each pair of which will serve as $R$-reciprocal congruences for a net on a surface and in general for only one.

\section{GREEN RECIPROCAL LINES IN RELATION $R$}

Suppose we have a pair of lines in relation $R$ with respect to the net (3). These lines may be obtained by joining the points $\bar{r}$ and $\bar{s}$ defined by

$$
\bar{r}=y_{\alpha}-\lambda^{\prime} y, \bar{s}=y_{\beta}-\mu^{\prime} y,
$$

and the point $y$ to the point $\bar{z}$ defined by

$$
\bar{z}=y_{\alpha \beta}-\mu^{\prime} y_{\alpha}-\lambda^{\prime} y_{\beta} .
$$

By methods similar to those used in $\$ 3$, we find that if these lines are to be Green reciprocal lines we must have

$$
\begin{aligned}
& 2 a^{\prime} \theta-\omega_{u}-\phi_{v} \frac{\partial(\theta \omega)}{\partial \alpha}-\left(\omega \mu^{\prime} \psi_{u}+\lambda^{\prime} \phi_{v}\right)(\theta \omega-1)=\left(\lambda^{\prime} \phi_{v}-\omega \mu^{\prime} \psi_{u}\right)(\theta \omega+1), \\
& 2 b \omega-\theta_{v}-\psi_{u} \frac{\partial(\theta \omega)}{\partial \beta}-\left(\theta \lambda^{\prime} \phi_{v}+\mu^{\prime} \psi_{u}\right)(\theta \omega-1)=\left(\mu^{\prime} \psi_{u}-\theta \lambda^{\prime} \phi_{v}\right)(\theta \omega+1) .
\end{aligned}
$$

Since these equations may be solved uniquely for $\lambda^{\prime}$ and $\mu^{\prime}$ we may state that associated with every non-conjugate, non-asymptotic net of curves on a nondevelopable surface there is one and only one pair of Green reciprocal congruences that are in relation $R$ with respect to the net. 


\section{THE RAY AND AXIS OF A POINT}

The line joining the two points

$$
\rho=y_{\alpha}-\bar{\gamma} y, \quad \sigma=y_{\beta}-\bar{\beta}^{\prime} y
$$

has been called the ray of the point $y$ with respect to the net*. The line joining $y$ to the point

$$
\zeta=y_{\alpha \beta}-\bar{\beta}^{\prime} y_{\alpha}-\bar{\gamma} y_{\beta}
$$

is called the axis of the point $y$ with respect to the net. $\dagger$ Let us find the condition on the net in order that the ray (axis) coincide with an arbitrary line lying in the tangent plane at $y$ (protruding from the surface at $y$ ).

Let the given arbitrary line lying in the tangent plane at $y$ join the two points

$$
r=y_{u}-\lambda y, \quad s=y_{v}-\mu y .
$$

If we impose the ccndition that this line coincides with the line determined by $\rho$ and $\sigma$ defined by (11) we find it necessary and sufficient that $\theta$ and $1 / \omega$ are solutions of

$$
\frac{\partial F}{\partial u}-F \frac{\partial F}{\partial v}=2 a^{\prime} F^{3}-2 \mu F^{2}-2 \lambda F+2 b .
$$

The net (3) will have the line (24) as ray.

Any line protruding from the surface at $y$ joins $y$ to the point

$$
z=y_{u v}-\mu y_{u}-\lambda y_{v} .
$$

If we impose the condition that this line coincide with the axis of $y$ determined by $y$ and $Z$ of (11) we find it necessary and sufficient that $\theta$ and $1 / \omega$ are any two solutions of the differential equation $\ddagger$

$$
\frac{\partial F}{\partial u}+F \frac{\partial F}{\partial v}=-2 a^{\prime} F^{3}+2 \mu F^{2}-2 \lambda F+2 b .
$$

Then the net (3) will have the arbitrary line $y z$ as axis. In that case the curves $C_{\alpha}, C_{\beta}$ are union curves of the congruence of lines $y z$.

* Green, Nets, p. 232 and footnote.

$\dagger$ Ibid., p. 224 and footnote.

$\ddagger$ Equations similar to (25) and (26) were obtained by E. Bompiani in a paper Sistemi coniugati e sistemi assiali di linee sopra una superficie dello spazio ordinario, Bollettino della Unione Matematica Italiana, vol. 3 (1924). In this paper Bompiani considers the problem of determining among the system of union curves of a given congruence two one-parameter families of curves forming a conjugate net. 
Let now the ray and axis of the point $y$ with respect to the net (3) coincide with a pair of arbitrary Green reciprocal lines. Then equations (25) and (26) will hold simultaneously. If we add and subtract these equations in the proper order we obtain the two equivalent ones

$$
F_{u}=-2 \lambda F+2 b, \quad F_{v}=2 \mu F-2 a^{\prime} F^{2} .
$$

A necessary and sufficient condition that equations (27) have a solution is that

$$
\frac{\partial}{\partial v}(b-\lambda F)=\frac{\partial}{\partial u}\left(\mu F-a^{\prime} F^{2}\right)
$$

be satisfied by such a solution. We may write this equation in the form

$$
\left(a_{u}^{\prime}-2 a^{\prime} \lambda\right) F^{2}+\left(4 a^{\prime} b-\lambda_{v}-\mu_{u}\right) F+\left(b_{v}-2 b \mu\right)=0 .
$$

Two possibilities arise:

1. Equation (28) may be an identity in $F$.

2. The quadratic may be solved for $F$.

In Case 1 let us assume that the surface is not ruled; we have therefore

$$
\lambda=\frac{a_{u}^{\prime}}{2 a^{\prime}}, \quad \mu=\frac{b_{v}}{2 b}, \quad 4 a^{\prime} b-\lambda_{v}-\mu_{u}=0 .
$$

The values for $\lambda$ and $\mu$ show that the assigned pair of Green reciprocal congruences is not arbitrary; they must be the directrix congruences of the first and second kind. The third equation of condition shows that the surface is also restricted. This condition may be reduced to the form

$$
\frac{\partial^{2}}{\partial u \partial v} \log \left(a^{\prime} b\right)=8 a^{\prime} b \text {. }
$$

If we put $\phi=\log \left(a^{\prime} b\right)$, equation (30) may be written

$$
\frac{\partial^{2} \phi}{\partial u \partial v}=8 e^{\phi} .
$$

The solution of (31) determines the function $\phi$ and hence a class of surfaces for which (30) holds. For each solution $\phi$, the function $F$ may be obtained by a quadrature from (27). Any two solutions $F_{1}\left(u, v, c_{1}\right), F_{2}\left(u, v, c_{2}\right) d e-$ termine a net such that the ray and axis of the point $y$ with respect to the net are the directrices of the first and second kinds.

In Case 2 we may solve the quadratic in $F$. Unless the roots are distinct, non-zero and finite, no net exists with the desired property. If we differentiate (28) with respect to $u$ and $v$ and use (27) we find that $F$ must satisfy a second 
quadratic in $F$ and a cubic in $F$. Imposing the condition that these quadratics have the same roots and that these roots are two of the roots of the cubic, we find that $\lambda$ and $\mu$ must satisfy equations of the form

$$
\begin{aligned}
\lambda_{v v}+\mu_{u v} & =G_{1}\left(\lambda_{u}, \lambda_{v}, \mu_{u}, \mu_{v}, \lambda, \mu, a^{\prime}, b\right), \\
\lambda_{u v}+\mu_{u u} & =G_{2}\left(\lambda_{u}, \lambda_{v}, \mu_{u}, \mu_{v}, \lambda, \mu, a^{\prime}, b\right), \\
0 & =G_{3}\left(\lambda_{u}, \lambda_{v}, \mu_{u}, \mu_{v}, \lambda, \mu, a^{\prime}, b\right), \\
0 & =G_{4}\left(\lambda_{u}, \lambda_{v}, \mu_{u}, \mu_{v}, \lambda, \mu, a^{\prime}, b\right) .
\end{aligned}
$$

The integrability conditions of (32) restrict the surface $S_{y}$ to be of a certain type. For surfaces of this type there exist Green reciprocal lines which will serve for the ray and axis of $y$ with respect to a net determined by the roots of (28).

\section{Pairs of CONJugate Nets}

Let us now consider the one-parameter family of curves

$$
\theta d u-d v=0 \text {. }
$$

The direction conjugate to the direction defined by (33) is defined by

$$
\theta d u+d v=0 \text {. }
$$

Let us consider also the family of conjugate nets

$$
\theta^{2} h^{2} d u^{2}-d v^{2}=0,
$$

where $h$ is an arbitrary constant but not zero. Wilczynski* has called such a one-parameter family of conjugate nets a pencil of conjugate nets. Wilczynski has also shown $\dagger$ that as this arbitrary net varies over all the nets of the pencil, the locus of the focal points of the tangents to the curves of the net at the point $y$ is a cubic curve. The equation of this curve referred to the triangle $y, y_{u}, y_{v}$ is

$$
C_{\theta} \equiv x_{1} x_{2} x_{3}+b x_{2}^{8}+a^{\prime} x_{3}^{3}-\frac{\theta_{u}}{2 \theta} x_{2}^{2} x_{3}+\frac{\theta_{v}}{2 \theta} x_{2} x_{3}^{2}=0 .
$$

Similarly the locus of the focal points of the tangents to the curves of the pencil

$$
\omega^{2} k^{2} d v^{2}-d u^{2}=0
$$

* E. J. Wilczynski, Geometrical significance of isothermal conjugacy of a net of curves, American Journal of Mathematics, vol. 42 (1920), p. 217.

† E. J. Wilczynski, Oral Communication to the American Mathematical Society, December, 1920, cited as W(1920); Lane, Nets, p. 290. 
is the cubic $C_{\omega}$ whose equation is

$$
C_{\omega} \equiv x_{1} x_{2} x_{3}+b x_{2}^{3}+a^{\prime} x_{3}^{3}+\frac{\omega_{u}}{2 \omega} x_{2}^{2} x_{3}-\frac{\omega_{v}}{2 \omega} x_{2} x_{3}^{3}=0 .
$$

The two curves $C_{\theta}$ and $C_{\omega}$ coincide if and only if $\theta \omega=$ const., that is, if and only if at every point of the surface the tangents to the net (3) form with the asymptotic tangents a constant cross ratio.*

If $C_{\theta}$ and $C_{\omega}$ are not identical, they intersect in nine points, the point $y$ counting as eight points and one other point $P$. This ninth point lies on the line

$$
(\theta \omega)_{u} x_{2}-(\theta \omega)_{v} x_{3}=0 .
$$

The nodal cubic $C_{\theta}$ has three inflection points. The line on which these points lie has been called the flex-ray of the point $y$ with respect to the net (35). The equation of this line referred to the triangle $y, y_{u}, y_{v}$ is

$$
x_{1}-\frac{\theta_{u}}{2 \theta} x_{2}+\frac{\theta_{v}}{2 \theta} x_{3}=0 \text {. }
$$

Similarly the flex ray of the point $y$ with respect to the net (37) is the line whose equation is

$$
x_{1}+\frac{\omega_{u}}{2 \omega} x_{2}-\frac{\omega_{v}}{2 \omega} x_{3}=0 .
$$

If $C_{\theta}$ and $C_{\omega}$ do not coincide, the flex rays (40) and (41) intersect in a point which lies on the line

$$
(\theta \omega)_{u} x_{2}-(\theta \omega)_{v} x_{3}=0 .
$$

Hence the ninth point of intersection of the ray-point cubics of two conjugate nets, the intersection of the two flex rays and the point $y$ are collinear.

The envelope of the osculating planes of the curves of the pencil (35) has been called the axis cone. $\dagger$ Wilczynski has shown that this surface is a cone of class three and that the cone is the space dual of the nodal cubic. The equation of the axis cone with respect to the net (35) in plane coordinates referred to the tetrahedron $y, y_{u}, y_{v}, y_{u v}$ is

$$
K_{\theta} \equiv u_{1} u_{2} u_{3}+a^{\prime} u_{2}^{3}+b u_{3}^{3}-\frac{\theta_{v}}{2 \theta} u_{2}^{2} u_{3}+\frac{\theta_{u}}{2 \theta} u_{2} u_{3}^{2}=0 .
$$

\footnotetext{
* If $\theta \omega=$ const. the nets $\theta^{2} d u^{2}-d v^{2}=0, \omega^{2} d v^{2}-d u^{2}=0$ belong to the same pencil of conjugate nets, and hence they determine the same ray-point cubic (Lane, Nets, p. 290).

† W (1920); Lane, Nets, p. 292.
} 
Similarly the axis cone of $y$ with respect to the net (37) has the equation

$$
K_{\omega} \equiv u_{1} u_{2} u_{3}+a^{\prime} u_{2}^{3}+b u_{3}^{3}+\frac{\omega_{v}}{2 \omega} u_{2}^{2} u_{3}-\frac{\omega_{u}}{2 \omega} u_{2} u_{3}^{2}=0 .
$$

If $\theta \omega$ is not constant, then $K_{\theta}$ and $K_{\omega}$ have nine common tangent planes, eight being accounted for at $y$. The ninth common tangent plane $\pi$ passes through the point

$$
(\theta \omega)_{v} u_{2}-(\theta \omega)_{u} u_{3}=0 .
$$

Each of the two cones $K_{\theta}$ and $K_{\omega}$ has three cusp axes which intersect in a line called by Wilczynski* the cusp axis of $y$. The plane of these two lines also passes through the point (42). Hence the ninth common tangent plane of the axis cones of two conjugate nets, the plane of the cusp axes of the cones, and the tangent plane to $S_{y}$ at $y$ intersect in a line. The equation of this line is

$$
(\theta \omega)_{u} x_{2}+(\theta \omega)_{v} x_{3}=0, \quad x_{4}=0 .
$$

The two lines (39) and (43) are the tangents to the curves of a unique conjugate net whose differential equation is

$$
(\theta \omega)_{u}^{2} d u^{2}-(\theta \omega)_{v}^{2} d v^{2}=0 .
$$

The line (43) is the tangent to the curve

$$
\phi(u, v)=\theta \omega=\text { const. }
$$

The family of curves defined by (45) is such that the cross ratio of the tangents to the curves of the net (3) and the asymptotic tangents is the same constant for points along the individual curves of the family.

We may associate a third cubic curve with the net (3) by means of the associate conjugate net $\dagger$ of the net (3). The differential equation of this net is readily seen to be

$$
\theta d u^{2}-\omega d v^{2}=0 .
$$

The ray-point cubic of the point $y$ with respect to the net (46) has the equation

* W (1920); Lane, Nets, p. 292.

† The associate conjugate net of a net has been defined by Green for any net on a surface as the net the tangents to whose curves are the double rays of the involution determined by the tangents to the curves of the given net and the asymptotic tangents. 


$$
\begin{aligned}
C_{\theta \omega} \equiv x_{1} x_{2} x_{3}+b x_{2}{ }^{8}+a^{\prime} x_{3}{ }^{8} & -\frac{1}{4}\left(\frac{\theta_{u}}{\theta}-\frac{\omega_{u}}{\omega}\right) x_{2}^{2} x_{8} \\
& +\frac{1}{4}\left(\frac{\theta_{v}}{\theta}-\frac{\omega_{u}}{\omega}\right) x_{2} x_{3}^{2}=0 .
\end{aligned}
$$

The flex ray of the point $y$ with respect to $C_{\theta \omega}$ has the equation

$$
x_{1}-\frac{1}{4}\left(\frac{\theta_{u}}{\theta}-\frac{\omega_{u}}{\omega}\right) x_{2}+\frac{1}{4}\left(\frac{\theta_{v}}{\theta}-\frac{\omega_{v}}{\omega}\right) x_{3}=0 .
$$

The cubic $C_{\theta \omega}$ is one of the curves of the pencil determined by $C_{\theta}$ and $C_{\omega}$ and the line (47) is a line of the pencil determined by the flex rays (40) and (41). Hence the ninth point of intersection of the cubics $C_{\theta}, C_{\omega}, C_{\theta \omega}$ and the intersections of the three flex rays are collinear with $y$. As a matter of fact any curve of the pencil determined by $C_{\theta}$ and $C_{\omega}$ has a flex ray which is one of the lines of the pencil determined by the flex rays of $C_{\theta}$ and $C_{\omega}$. The Hessians of the curves of the pencil determined by $C_{\theta}$ and $C_{\omega}$ have their common ninth point on the line (41). The corresponding dual theorems are also true.

\section{A ONE-PARAMETER FAMILY OF CURVES}

Let us now consider the case in which the given net is composed of an arbitrary one-parameter family of curves and one of the families of asymptotic curves. Let the given net be

$$
(\theta d u-d v) d v=0 .
$$

We may make the net (48) parametric by the transformation

$$
\alpha=\phi(u, v), \quad \beta=v, \quad \phi_{u}=-\theta \phi_{v} .
$$

From (49) we derive the differentiation formulas

$$
y_{\alpha}=-\frac{y_{u}}{\theta \phi_{v}}, \quad y_{\beta}=\frac{y_{u}+\theta y_{v}}{\theta},
$$

from which we obtain

$$
y_{\alpha \beta}=-\frac{1}{\theta^{3} \phi_{v}}\left(\theta^{2} y_{u v}-\theta_{u} y_{u}-2 b \theta y_{v}-f \theta y\right) .
$$

Consider the line $l$ joining the points $\bar{r}$ and $\bar{s}$ defined by

$$
\bar{r}=y_{\alpha}-\bar{\lambda} y, \quad \bar{s}=y_{\beta}-\bar{\mu} y .
$$


The line $l^{\prime}$ in relation $R$ to $l$ with respect to the net (48) joins $y$ to the point*

$$
\bar{z}=y_{\alpha \beta}-\bar{\mu} y_{\alpha}-\bar{\lambda} y_{\beta} .
$$

If we use (50) and (51), (52) and (53) may be written

$$
\begin{aligned}
& -\theta \phi_{v} \bar{r}=y_{u}-\lambda y, \quad \theta \bar{s}=y_{u}+\theta y_{v}-\bar{\mu} \theta y, \\
& -\theta \phi_{v} \bar{z}=y_{u v}-\frac{1}{\theta}\left(\frac{\theta_{u}}{\theta}+\theta \bar{\mu}+\lambda\right) y_{u}-\left(\frac{2 b}{\theta}+\lambda\right) y_{v},
\end{aligned}
$$

wherein

$$
\lambda=-\theta \phi_{v} \lambda^{\prime}
$$

The line $l$ intersects the asymptotic tangents in the points

$$
r=y_{u}-\lambda y, \quad s=y_{v}-\frac{1}{\theta}\left(\theta \mu^{\prime}-\lambda\right) y .
$$

The lines $l$ and $l^{\prime}$ will therefore be Green reciprocal lines if, and only if, $b=0$ and $\lambda=-\theta_{u} /(2 \theta)$. Hence to each curve of $a$ one-parameter family through $y$ on a ruled surface $S_{y}$ there corresponds one and only one point $r$ on $S_{y}$ through which lines may be drawn whose Green reciprocal lines are in relation $R$ with respect to the net composed of the given one-parameter family and the generators of $S_{y}$. This point lies on the generator through $y$. Moreover, as may be shown, the lines of this pencil have coplanar Green reciprocal lines.

This theorem suggests that certain interesting results might be obtained if the point $y$ were a flecnode or complex point, and the given curve through $y$ the flecnode or complex curve. These investigations are beyond the scope of this paper and we will leave them for some future time.

* Grove, Nets, p. 493.

Michigan State College, East Lansing, Mich. 